\title{
Facile Preparation of a Hairpin DNA-Gold Nanoparticle Monoconjugate with a Single-Dye Molecule and Lactobionic Acid as Targeting Ligand
}

\author{
Akane MukaIda,* Rihito AdaChI, ${ }^{*}$ Yoshitsugu AkiYama, ${ }^{* * \dagger}$ and Masao Kamimura* \\ *Department of Materials Science and Technology, Graduate School of Industrial Science and Technology, \\ Tokyo University of Science, 6-3-1 Niijuku, Katsushika, Tokyo 125-8585, Japan \\ **Faculty of Industrial Science and Technology, Tokyo University of Science, 6-3-1 Niijuku, Katsushika, \\ Tokyo 125-8585, Japan
}

\begin{abstract}
We established a new design for a single molecular beacon-conjugated gold nanoparticle, named monoMB-GNP, which showed enhanced fluorescence emission only in the presence of the complementary DNA sequence. MonoMB-GNP also showed no apparent toxicity to NIH/3T3 cells at $1 \mathrm{nM}$, as determined by the water-soluble tetrazolium assay. Importantly, the lactobionic acid was successfully modified on the surface of monoMB-GNP. The proposed nanoparticle has prospects for use in several applications for targetable molecular beacon strategies.
\end{abstract}

Keywords Gold nanoparticle, molecular beacon, monoconjugation, targeting

(Received November 30, 2020; Accepted February 18, 2021; Advance Publication Released Online by J-STAGE March 5, 2021)

\section{Introduction}

Fluorescent probes that can detect and target nucleic acids (DNA and RNA) with high sensitivity have become indispensable tools in biological research. Among them, a stem-loop structurebased molecular beacon (MB) comprising a fluorescent dye and a quencher at each terminal, respectively, enables sequencespecific fluorescence detection in living cells in real time when it forms a duplex with the target nucleic acid. ${ }^{1-4}$ This inherent signal transduction mechanism of MBs can facilitate the analysis of target oligonucleotides without the separation of unbound fluorescent probes. In addition, cell-specific biomolecules functionalized with MBs allow real-time detection of intracellular mRNA in living cells in vitro. ${ }^{5}$ However, there are several concerns related to the practical use of MB-based technologies, particularly for in vivo applications, due to low blood retention and cell uptake efficiency.

Recently, gold nanoparticles (GNPs), which act as quenchers, were modified with a highly dense MB harboring a stem-loop structure on the surface to obtain MB-modified GNP (MBGNP), thereby achieving efficient cell uptake in vitro ${ }^{6-9}$ and in vivo. ${ }^{10}$ Generally, MB-GNPs facilitate the binding of DNA to the GNP due to the strong affinity between thiol groups and gold. Hence, GNP with MBs densely covering its surface result in a significant increase in fluorescence intensity only in the presence of the targeted DNA. However, MB-GNPs with a high-density MB brush layer did not have sufficient space for binding biomolecules possessing strong affinity to specific cells. In contrast, a monofunctional gold cluster (GC, $\sim 1.4 \mathrm{~nm}$ diameter) covered with phosphine ligands possessing a maleimide group was covalently modified with thiol-terminated

† To whom correspondence should be addressed.

E-mail: yoshitsugu.akiyama@rs.tus.ac.jp
MBs, resulting in the formation of monoMB-GC-containing fluorescent dye molecules, such as fluorescein, rhodamine, and cyanine-5 (Cy5). ${ }^{11,12}$ However, bigger particles, of at least $5 \mathrm{~nm}$ in diameter, are generally used for in vivo applications since small GNPs are more rapidly eliminated via renal filtration. ${ }^{13}$ Moreover, in the case of $\mathrm{GC}$, the introduction of ligands that increase the affinity for target cells seems to be limited. Considering these setbacks, the establishment of a versatile and simple methodology for realizing in vivo fluorescence detection of nucleic acids in live target cells still represents a challenge.

Here, we report a versatile and simple methodology to obtain MB-attached GNP monoconjugates (monoMB-GNP) via the thiol-gold interaction, followed by further modification of lactobionic acid (LBA) as model targeting ligand. These monoMB-GNPs possessing LBA may enable quantitative nucleic acid detection in specific cells.

\section{Experimental}

\section{Reagents and chemicals}

All chemicals were used without further purification and were purchased from commercial sources as indicated: bis( $p$-sulfonatophenyl)phenylphosphine (BSPP) dehydrate dipotassium salt (Sigma-Aldrich, USA), tris-borate-EDTA buffer (Nippon Gene, Japan), agarose L03 for electrophoresis (Takara Bio, Japan), GNPs with a nominal diameter of $5 \mathrm{~nm}$ (BBI Solutions, UK), chemically synthesized DNA strands with and without modifications (Tsukuba Oligo Service, Japan), Cell Counting Kit-8 (CCK-8) for cytotoxicity assays (Dojindo, Japan), and frozen stocks of mouse fibroblast NIH/3T3 cells (RIKEN Bioresource Center, Japan). All other chemicals used were purchased from FUJIFILM Wako Pure Chemical Corporation, Japan. Ultrapure water $(>18 \mathrm{M} \Omega \cdot \mathrm{cm})$ was prepared using a Milli-Q water purification system (Millipore, USA), which was 

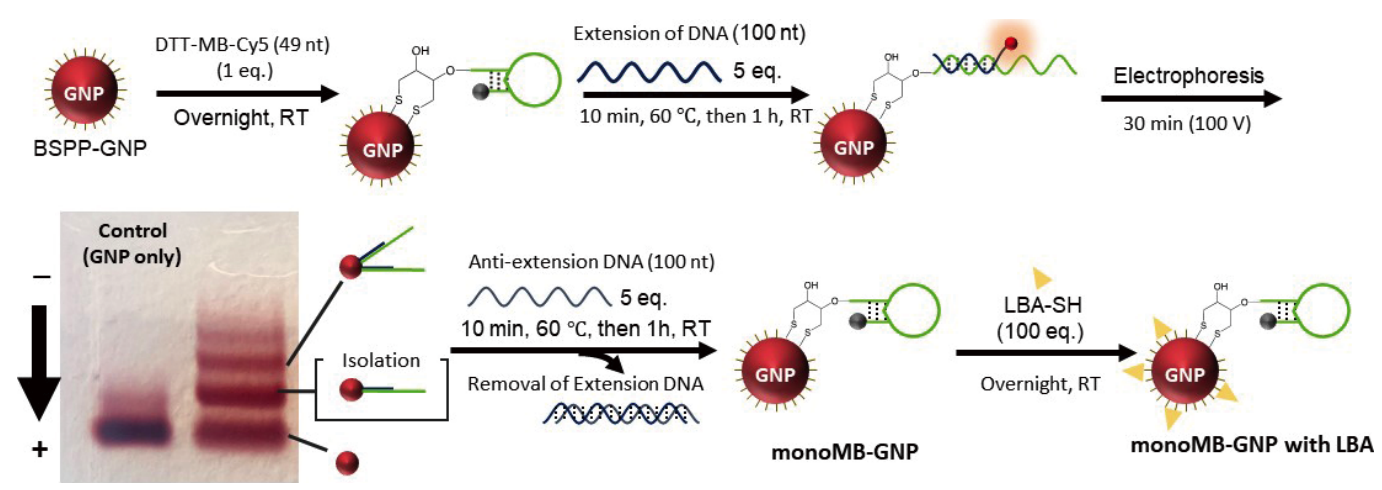

Fig. 1 Schematic illustration of monoMB-GNP, which provides a space for introducing cell-specific biomolecules (LBA) on the surfaces of GNP. BSPP, bis( $p$-sulfonatophenyl)phenylphosphine dihydrate dipotassium salt; LBA-SH, of lactobionic acid containing a thiol group; GNP, gold nanoparticle; MB, molecular beacon; monoMB-GNP, MB-attached GNP monoconjugates; NT, nucleotides, RT, room temperature.

used for all experiments. The DNA concentration was determined by measuring the absorbance at $260 \mathrm{~nm}$ with a UV-vis spectrophotometer (V650; JASCO, Japan). Fluorescence experiments were performed in a micro fluorescence cell with a path length of $1.0 \mathrm{~cm}$ using a spectrofluorophotometer (RF5300PC, SHIMADZU, Japan). The DNA sequences used in this study are shown in Table S1 (Supporting Information).

Further experimental details, including monoMB-GNP preparation, fluorescent characterization of the monoMB-GNP, and the cytotoxicity test of monoMB-GNP in NIH/3T3 cells, are provided in the Supporting Information.

\section{Results and Discussion}

MB-GNP can show a strong surface plasmon resonance (SPR) band and the ability to quench dyes efficiently. However, these properties are markedly affected by several factors, such as the GNP-dye distance, GNP size, and dye type. Therefore, the MB used in this study was carefully designed. As shown in Fig. 1 and Fig. S1 (Supporting Information), a Cy5 was introduced at the 3 -terminal position in the $49 \mathrm{nt}$ MB containing the 7 base pairs for stem and $25 \mathrm{nt}$ for loop structures. In addition, a DTT group, which has stronger affinity than that of the monothiol group,${ }^{14}$ was bound to the gold surface at the $5^{\prime}$ position of the MB using a $10 \mathrm{nt}$ thymine base as linker. This MB strand possessing a DTT group at the $5^{\prime}$-position was also directly monoconjugated with a GNP (5 nm in diameter) for the formation of the monoMB-GNP. Thus, the Cy5 signal is quenched by fluorescence resonance energy transfer, while the fluorescence is emitted by increasing the distance between Cy5 and GNP when a sequence complementary to the loop structure is added.

To prepare monoMB-GNP, BSPP-stabilized GNP $(5 \mathrm{~nm})$ and DTT-MB-Cy5 were mixed at different molar ratios of MB to GNP and incubated overnight at room temperature. After adding 5 equiv. of extended strands $(100 \mathrm{nt})$ to the reaction mixture, separation was performed by agarose gel electrophoresis (3\% agarose, $100 \mathrm{~V}, 30 \mathrm{~min}) .^{15,16}$ Figure S2 (Supporting Information) shows that multiple red bands derived from the MB-GNPs were detected, revealing the highest formation efficiency of the monoconjugate at an MB:GNP ratio of 1 . This result indicated that the reaction was stoichiometric. Hence, BSPP-GNP and DTT-MB-Cy5 were mixed at 1:1 molar ratio

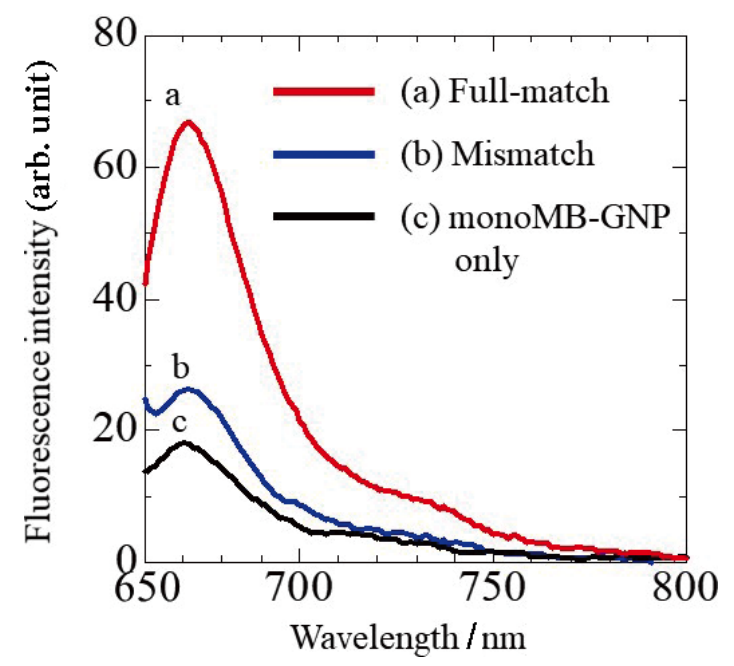

Fig. 2 Fluorescence spectra of molecular beacon-attached gold nanoparticles monoconjugates (monoMB-GNP; $333 \mathrm{nM}$ ) upon hybridization with (a) the fully matched DNA sequence, (b) mismatched DNA sequence, and (c) without DNA as a control in appropriate buffer solution, $\mathrm{pH} 7.4\left(\lambda_{\mathrm{ex}}=646 \mathrm{~nm}\right)$.

and allowed to react using the same procedure. Electrophoretic isolation from the agarose gel and several rounds of centrifugation resulted in the desired monoMB-GNP with a $10 \%$ yield, which was in good agreement with the conventional preparation of ssDNA-monoconjugated GNP. ${ }^{16}$ To our knowledge, this is the first report of DTT-terminated hairpin DNA with a single-dye molecule to be directly conjugated with GNP for monoMB-GNP preparation. More importantly, it can be expected that monoMB-GNP will find applications in the quantitative fluorescent detection of target nucleic acids due to avoidance of steric hindrance of DNA binding to monoMBGNP, as compared with densely MB-covered GNP.

Figure 2 shows the fluorescence spectra of monoMB-GNP with and without 10 equiv. of ssDNA (fully matched and mismatched) at room temperature. The emission of monoMBGNP with a mismatched sequence was not significantly different from that of the control sample (monoMB-GNP only) in the emission range, indicating the high quenching efficiency 


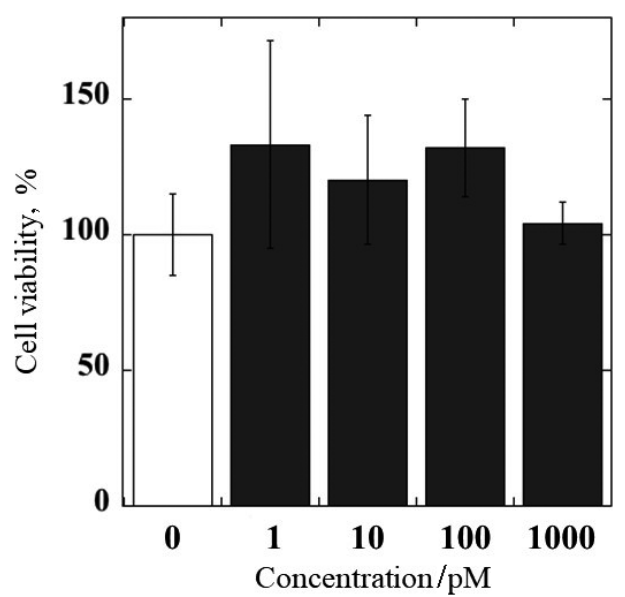

Fig. 3 Viability of NIH/3T3 cells with increasing concentrations of molecular beacon-attached gold nanoparticles monoconjugates (monoMB-GNPs). Cell viability was measured using the CCK-8 assay. Data are shown as mean \pm standard deviation $(n=3)$.

conferred by the Cy5 conjugate. There was a distinguishable difference in the fluorescence intensity ratio of the fully matched to mismatched sequences $\left(F_{\text {full-match }} / F_{\text {mismatch }}=2.4\right)$, indicating that sequence specificity can be detected at room temperature. This result suggested that the proper MB design with a suitable dye molecule and balance between the MB and GNP size led to enhanced fluorescence. Indeed, monoMB-GNP conjugated with fluorescein (FAM) at the end of the MB with similar and same-sized GNPs did not show such sequence-specific increase in fluorescence intensity (Fig. S3, Supporting Information).

To evaluate the molar ratio-dependent performance of monoMB-GNP, fluorescence experiments with varying concentrations of ssDNA were performed. Figure S4 (Supporting Information) shows the fluorescence intensity of monoMB-GNP in the presence of a fully matched sequence (full-match) at an excitation wavelength of $646 \mathrm{~nm}$ with a DNA/GNP ratio of 0.5 , 1,5 , and 10 in an appropriate buffer solution. The fluorescence enhancement was observed from an increase in the DNA:GNP ratio to 1 equiv. of full-match. Additionally, when the excess full-match altered from 1 to 10 equiv. in the DNA:GNP ratio was added, the fluorescence emission of monoMB-GNP retained 1 equiv. of full-match, indicating that monoMB-GNP emission can reach the maximum intensity with efficient emission enhancement. In a previous report, a $2.5-\mathrm{nm}$ diameter GNP mixed with $2-3$ equiv. of ssDNA possessing a fluorescent dye at the terminal exhibited approximately 10-times higher fluorescence emission in the presence of 4 equiv. of the target ssDNA. ${ }^{17}$ However, an unknown number of ssDNA on GNP and low colloidal stability of the fluorescent dye attached GNP without BSPP as a phosphine ligand would limit the quantitative characterization. Therefore, efficient recognition of full-match at 1:1 (DNA:GNP) ratio from monoMB-GNP with BSPP can be applicable for in vivo use.

To estimate cell damage against the monoMB-GNP, the cytotoxicity of monoMB-GNP was evaluated using the watersoluble tetrazolium assay. As shown in Fig. 3, NIH/3T3 cells treated with monoMB-GNP (up to $1 \mathrm{nM}$ ) for $2 \mathrm{~h}$ survived without noticeable toxicity. Considering that the particle concentration generally used in living cells is within 0.1 $1 \mathrm{nM},{ }^{18}$ this result indicates that monoMB-GNP may be safe for fluorescence imaging-based applications.

To introduce the specific cell-targeting ability into monoMB-
GNP, monoMB-GNP with a modification space of the surface was treated with galactose derivatives, which can recognize the asialoglycoprotein receptor (ASGP-R) overexpressed by hepatic carcinoma cells. ${ }^{19}$ Consequently, 100 equiv. of thiolated lactobionic acid (LBA-SH) ${ }^{20}$ was mixed with monoMB-GNP and incubated overnight at room temperature. Excess LBA-SH was removed by centrifugation and redispersed in appropriate buffer solution. By comparison with monoMB-GNP (without LBA-SH treatment), the monoMB-GNP modified with LBA-SH resulted in good recovery efficiency (89\%) characterized by UV-vis spectroscopy with absorbance at $520 \mathrm{~nm}$ derived from SPR of dispersed GNP (Fig. S5, Supporting Information). This result suggested that monoMB-GNP modified with LBA could be dispersed in an aqueous solution.

In conclusion, monoMB-GNP, which is a combination of $\mathrm{Cy} 5$ and GNPs in MB, achieved a sequence-specific increase in fluorescence intensity. Moreover, the addition of 1 equiv. of a fully matched sequence showed a stoichiometric-dependent increase in fluorescence. Furthermore, in vitro cell culture experiments further revealed that monoMB-GNP has low cytotoxicity. Treatment with LBA as a targetable moiety exhibited a high dispersion state after purification. Details of the LBA recognition ability of monoMB-GNP and the fluorescent properties of monoMB-GNP with LBA as a model ligand are underway and will be published elsewhere. Therefore, this monoMB-GNP takes advantage of (i) the simple modification of hairpin DNA with a single molecule of Cy5 through a DTT moiety at the terminal, (ii) expectation of quantitative fluorescence analysis due to the same concentration of GNP with MB, and (iii) the specific cell targeting from ligand attachment (LBA) on monoMB-GNP. Thus, monoMB-GNP has the potential to be used for in vitro assays in living cells as well as in vivo targetable fluorescent imaging by the introduction of ligands that can increase the cell affinity.

\section{Supporting Information}

This material is available free of charge on the Web at http:// www.jsac.or.jp/analsci/.

\section{References}

1. S. Tyagi and F. R. Kramer, Nat. Biotechnol., 1996, 14, 303.

2. X. Fang, X. Liu, S. Schuster, and W. Tan, J. Am. Chem. Soc., 1999, 121, 2921.

3. M. B. Baker, G. Bao, and C. D. Searles, Nucleic Acids Res., 2012, 40, e13.

4. D. Xiang, K. Zhai, Q. Sang, B. Shi, and X. Yang, Anal. Sci., 2017, 33, 275.

5. N. Nitin, P. J. Santangelo, G. Kim, S. Nie, and G. Bao, Nucleic Acids Res., 2004, 32, e58.

6. A. Jayagopal, K. C. Halfpenny, J. W. Perez, and D. W. Wright, J. Am. Chem. Soc., 2010, 132, 9789.

7. H. N. Joo and Y. J. Seo, Tetrahedron Lett., 2015, 56, 542.

8. Y. Ma, Z. Wang, M. Zhang, Z. Han, D. Chen, Q. Zhu, W. Gao, Z. Qian, and Y. Gu, Angew. Chem. Int. Ed., 2016, 55, 3304.

9. T. Xie, Z. Fan, R. Zhang, X. Tian, G. Han, Z. Liu, and Z. Zhang, Anal. Methods, 2020, 12, 2385.

10. M. I. Uddin, T. C. Kilburn, R. Yang, G. W. McCollum, D. W. Wright, and J. S. Penn, Mol. Pharmaceutics, 2018, 15, 5514.

11. B. Dubertret, M. Calame, and A. J. Libchaber, Nat. 
Biotechnol., 2001, 19, 365.

12. Y. Tian, L. Zhang, and L. Wang, Biotechnol. J., 2020, 15, 1800741.

13. M. Longmire, P. L. Choyke, and H. Kobayashi, Nanomedicine, 2008, 3, 703.

14. F. Li, H. Zhang, B. Dever, X.-F. Li, and X. C. Le, Bioconjugate Chem., 2013, 24, 1790.

15. D. Zanchet, C. M. Micheel, W. J. Parak, D. Gerion, and A. P. Alivisatos, Nano Lett., 2001, 1, 32.

16. Y. Akiyama, H. Shikagawa, N. Kanayama, T. Takarada, and
M. Maeda, Small, 2015, 11, 3153.

17. D. J. Maxwell, J. R. Taylor, and S. Nie, J. Am. Chem. Soc., 2002, 124, 9606.

18. D. S. Seferos, D. A. Giljohann, H. D. Hill, A. E. Prigodich, and C. A. Mirkin, J. Am. Chem. Soc., 2007, 129, 15477.

19. K. M. Kamruzzaman Selim, Y. S. Ha, S. J. Kim, Y. Chang, T. J. Kim, G. Ho Lee, and I. K. Kang, Biomaterials, 2007, 28,710 .

20. W. Chen, Y. Zou, F. Meng, R. Cheng, C. Deng, J. Feijen, and Z. Zhong, Biomacromolecules, 2014, 15, 900. 